\title{
Control of a Dual Fed Open End Winding SPMSM with a Floating Capacitor
}

\author{
$1^{\text {st }}$ Davide Minaglia \\ Danieli Automation S.P.A. \\ Genoa, IT \\ minagliad@danieli.it
}

\author{
$2^{\text {nd }}$ Luca Rovere \\ PEMC \\ University of Nottingham \\ Nottingham, UK \\ luca.rovere@nottingham.ac.uk
}

\author{
$5^{\text {th }}$ Sabino Pipolo \\ PEMC \\ University of Nottingham \\ Nottingham, UK \\ sabino.pipolo@nottingham.ac.uk
}

\author{
$3^{\text {rd }}$ Andrea Formentini \\ PEMC \\ University of Nottingham \\ Nottingham, UK \\ andrea.formentini@nottingham.ac.uk \\ $6^{\text {th }}$ Mario Marchesoni \\ DITEN \\ University of Genoa \\ Genoa, IT \\ marchesoni@unige.it
}

\author{
Computer and Biomedical Engineering \\ University of Pavia \\ Pavia, IT \\ riccardo.leuzzi@unipv.it
}

$4^{\text {th }}$ Riccardo Leuzzi
Department of Electrical,

$7^{\text {th }}$ Pericle Zanchetta

PEMC, Department of Electrical,

Computer and Biomedical Engineering

University of Nottingham, University of Pavia

Nottingham, UK, Pavia, IT

pericle.zanchetta@nottingham.ac.uk, pericle.zanchetta@unipv.it

\begin{abstract}
Surface Permanent Magnet Synchronous Motor (SPMSM) are not the first choice when a motor drive is required to operate over a wide speed range with an extended Constant Power Speed Range (CPSR). Nevertheless, SPMSMs offer high torque density, high efficiency simple manufacturing process and robustness which makes them an attractive alternative to Interior PMSMs. The Open End Winding (OEW) dual inverter topology, with one of the two Voltage Source Inverters (VSI) connected to a floating capacitor (FC), has shown to be capable of significantly increasing the operating speed range and CPSR of the SPMSM at the price of an increased controller complexity. In this paper, the analytical state space model of the OEW-FC SPMSM is derived, a state feedback controller is proposed and a possible tuning strategy is given to allow for high dynamics over the whole operating range and simplified controller design and tuning.

Index Terms-Open-End Winding Machine, Permanent Magnet Synchronous Motor, Dual-Fed, Floating Capacitor, StateSpace Control, Linear Quadratic Regulator.
\end{abstract}

\section{INTRODUCTION}

When an electric drive is required to operate over a wide speed range, Interior Permanent Magnet Synchronous Motors (IPMSMs) are generally preferred over SPMSM due to their lower flux and higher anisotropy. On the other hand, SPMSMs offer high torque density, high efficiency simple manufacturing process and robustness. To increase the speed range of SPMSMs drives the dual inverter topology has proven to be one of the most interesting and advantageous approaches. The star connected stator windings of the machine are opened allowing for the machine to be fed from both sides by standard two levels VSIs. The OEW topology allows to increment the machine phase voltage and therefore the operating range of the motor [1], [2]. If separate dc links are available, each VSI can be fed independently allowing for three level, five level or higher voltage output according to the two dc links voltage ratios [3]. If instead two dc links are not available, both the VSIs can be connected on a common dc link. This solution shows increased speed range and overall simplicity but allows for the circulation of additional common mode currents. Different modulation strategies which effectively eliminate the common mode voltage generated by the two VSIs have been proposed, still resulting in a reduction of the linear modulation range and consequently of the maximum speed for a given dc link [4], [5]. The dual inverter OEW topology most capable of significantly extending the machine operating range is the one where the second VSI has its $\mathrm{dc}$ link connected to a FC [6]-[8]. The operating speed range and the CPSR are greatly extended thanks to the second VSI connected to a FC which provides for the reactive power required by the SPMSM. Therefore, the FC bridge acts as a power factor compensator for the battery connected VSI, which is then able to transfer the maximum active power over the whole speed range. In addition, the efficiency of the second VSI can be increased by properly controlling the FC voltage according to the system operating point [9], effectively resulting into reduced switching losses. The OEW- 


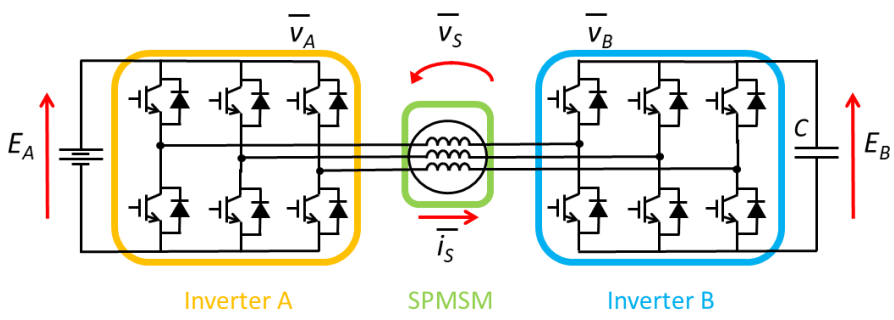

Fig. 1. Schematic diagram of the OEW SPMSM. The main VSI A is connected to the battery bank while VSI B is connected to a floating capacitor.

FC dual inverter topology provides a concrete alternative to the increment of the DC battery voltage, furthermore it allows to supply the machine with a multi-level PWM, increase the fault-tolerant capability and provides redundant spacevector combinations. The efficiency of the second VSI can be increased by properly controlling the FC voltage according to the system operating point [9], effectively resulting into reduced switching losses. Different control schemes have been proposed for the OEW-FC, in [10] a decoupled control strategy of the two VSIs has been developed. In [11] a Finite Set Model Predictive Control technique applied to a dual-active bridge inverter with FC has been presented while in [7] a control strategy aimed at keeping at unity the power factor of the battery connected VSI is proposed. In [8] the speed range extension allowed by the secondary VSI control has been studied but no detail is given on the controller design and tuning. The extended operating range comes at the price of increased controller complexity. In fact, achieving high performance control of the machine's dynamics and the FC voltage simultaneously has proven to be a challenging task due to the highly coupled dynamics of the two VSIs. As it can be seen from the SPMSM OEW-FC analytical model developed in this paper, the system's equations are non linear, therefore obtaining a transfer function to be used for the tuning of the controller is not straightforward. The main contribution of this work is the formulation of the plant equations in a state space form in order to allow for linearisation and synthesis of the state feedback controller. Using a Linear Quadratic Regulator (LQR), which aims to minimize a user defined cost $\mathrm{J}$, the gains of the feedback controller can be computed.

\section{OEW-FC SPMSM MODEL}

The mathematical model of a SPMSM in the dq synchronous reference frame with the d-axis aligned with the excitation flux is presented in (1)

$$
\left\{\begin{array}{l}
\bar{v}_{s}=\bar{v}_{A}-\bar{v}_{B} \\
v_{d}=R_{s} i_{d}+\frac{d \varphi_{d}}{d t}-w_{e} \varphi_{q} \\
v_{q}=R_{s} i_{q}+\frac{d \varphi_{q}}{d t}+w_{e} \varphi_{d} \\
\varphi_{d}=L_{s} i_{d}+\lambda_{m} \\
\varphi_{q}=L_{s} i_{q} \\
T_{e}=\frac{3}{2} p \lambda_{m} i_{q}
\end{array}\right.
$$

where according to Fig. $1 \bar{v}_{s}$ are the SPMSM stator voltages while $\bar{v}_{A}$ and $\bar{v}_{B}$ are the VSI A and VSI B voltages respectively. $v_{d}, v_{q}$ and $\varphi_{d}, \varphi_{q}$ are the machine dq voltages and fluxes, while $i_{d}$ and $i_{q}$ and are the machine dq currents. $w_{e}$ is the machine electrical pulsation, $T_{e}$ is the electromagnetic torque, $p$ are the pole pairs number and $\lambda_{m}$ is the flux generated by the permanent magnet. The matrix form of the machine equations is shown in (2), which will be useful for the development of the state space system model.

$\left[\begin{array}{l}v_{d} \\ v_{q}\end{array}\right]=\left[\begin{array}{cc}R_{s} & -w_{e} L_{s} \\ w_{e} L_{s} & R_{s}\end{array}\right]\left[\begin{array}{l}i_{d} \\ i_{q}\end{array}\right]+\left[\begin{array}{cc}L_{s} & 0 \\ 0 & L_{s}\end{array}\right] \frac{d}{d t}\left[\begin{array}{c}i_{d} \\ i_{q}\end{array}\right]+\left[\begin{array}{c}0 \\ w_{e} \lambda_{m}\end{array}\right]$

The voltage equation that describes the VSI B FC dynamics can be derived as follows

$$
\frac{d}{d t} E_{B}=\frac{i_{C}}{C}
$$

where $i_{C}$ is the current flowing through the capacitor and $C$ is its capacitance. The dq reference frame duty cycles of the two VSIs when a spacevector PWM with third harmonic injection is used are defined as

$$
\delta_{d}^{A}=\frac{\sqrt{3} v_{d}^{A}}{E_{A}} \quad \delta_{q}^{A}=\frac{\sqrt{3} v_{q}^{A}}{E_{A}} \quad \delta_{d}^{B}=\frac{\sqrt{3} v_{d}^{B}}{E_{B}} \quad \delta_{q}^{B}=\frac{\sqrt{3} v_{q}^{B}}{E_{B}}
$$

Combining (4) with (3), the following expression is obtained

$$
\frac{d}{d t} E_{B}=\frac{3}{4 C}\left(\delta_{d}^{B} i_{d}+\delta_{q}^{B} i_{q}\right)
$$

It can be observed that (5) contains the non-linearity represented by the product of the dq currents with the duty cycles, i.e. the control actions. Equation (5) corresponds to the same problem of control of an Active Front End (AFE) converter, characterized has a multi-input-multi-output non-linear structure [12]. Having defined the equations that describe the SPMSM and FC dynamics, (2) and (5) can be rearranged to obtain the OEW-FC system model in state-space form.

\section{A. OEW-FC SPMSM Reactive Power Compensation}

The role of the FC is to provide the reactive power needed by the machine according to its operating point, in this way VSI A can provide exclusively the active power required. In other words, VSC B voltage can be controlled to use the second inverter as a series compensator, the load impedance seen by VSI A is made of the motor impedance plus the series equivalent impedance of VSC B which can be changed by controlling $\bar{v}_{B}$ to act as a capacitive or inductive load. The active power delivered by VSI A consists in the Joule losses, the iron losses and the mechanical power delivered to the motor. On the other hand, the active power transferred to VSI B consists in the power necessary to charge the FC, considering that the VSI $\mathrm{B}$ converter losses ar negligible they are not considered during the analytical model formulation. The voltage constraints for $\bar{v}_{A}$ and $\bar{v}_{B}$ according to the previous mode of operation are obtained by solving the following set of equations: 


$$
\left\{\begin{array}{l}
P_{B}=\frac{3}{2}\left(v_{A}^{d} i_{d}+v_{B}^{q} i_{q}\right)=0 \\
Q_{A}=\frac{3}{2}\left(j w_{e} \bar{\varphi}_{s} \cdot j \bar{i}_{s}+\bar{v}_{B} \cdot \bar{i}_{s}\right)=0
\end{array}\right.
$$

where the voltage drop due to the stator resistance has been neglected being generally smaller than the voltage drop due to the machine winding inductance. The resolution of (6) leads to the following VSI A and B voltages

$$
\left\{\begin{array}{l}
v_{A}^{d}=\frac{i_{d} i_{q} w_{e} \lambda_{m}}{\left|\bar{s}_{s}\right|^{2}} \\
v_{A}^{q}=\frac{i_{q}^{2} w_{e} \lambda_{m}}{\left|\bar{i}_{s}\right|^{2}} \\
v_{B}^{d}=\frac{i_{q} w_{e}\left(L_{s}\left|\bar{s}_{s}\right|^{2}+\lambda_{m} i_{d}\right)}{\left|\bar{i}_{s}\right|^{2}} \\
v_{B}^{q}=\frac{-i_{d} w_{e}\left(\left.\left.L_{s}\right|_{s}\right|^{2}+\lambda_{m} i_{d}\right)}{\left|\bar{i}_{s}\right|^{2}}
\end{array}\right.
$$

The theoretical voltages calculated in (7) are the optimal ones that need to be applied to the machine in order to keep the reactive power $Q_{A}$ of VSI A to zero. Furthermore, observing that

$$
Q_{A}=Q_{S}+Q_{B}=0
$$

by imposing the machine voltages (7) the total reactive power required by the machine $Q_{S}$ would be provided by VSI $\mathrm{B}$. The OEW-FC drive speed range and its torque characteristic have been thoroughly discussed in [8] where it has been shown that the dual inverter drive with a FC is able to extend the speed rage of the SPMSM according to the dc link voltages ratio as

$$
\frac{\omega_{O E W-F C}^{\max }}{\omega_{S I}^{\max }}=1+\frac{E_{B}^{\max }}{E_{A}}
$$

where $\omega_{O E W-F C}^{\max }$ and $\omega_{S I}^{\max }$ are the maximum speed achievable with an OEW-FC and a standard 2L-VSI drive respectively. The torque characteristic can be determined by imposing the the voltage and current capability of the inverter and the SPMSM. The voltage limits have defined in (4) while the value of the admissible stator current $I_{\max }$ corresponds to the nominal thermal current of the machine at steady state or the nominal current of the inverters in transient overload conditions. It has to be noted that, although VSI B is only delivering reactive power to the motor, a very small amount of real power is needed to balance the losses in the inverter and FC.

\section{DUAL-INVERTER VA ANALYSIS}

Regarding the voltage and current rating of the OEW-FC topology we can observe from (9) that the maximum speed of the SPMSM in an OEW-FC configuration depends on the floating capacitor maximum allowed voltage. Therefore, according to the requirements of the application, the voltage rating of VSI B can be sized differently. The size of the FC has a strong impact on the size and cost of the OEW drive, the dimensioning of the FC depends on the voltage rating, the accepted voltage ripple and the peak current through it. Considering the capacitor ESR and the ripple rms current an electrical and thermal sizing can be carried out. Regarding the
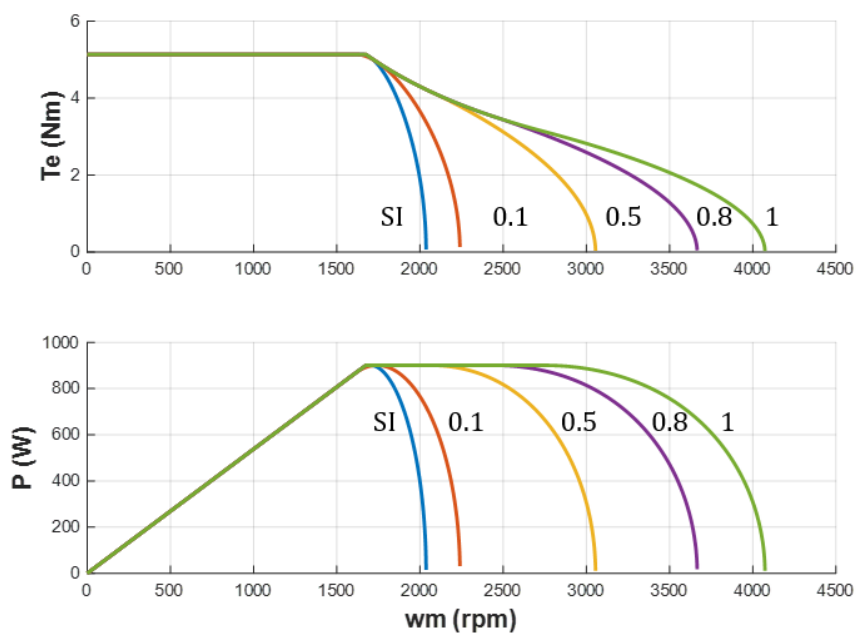

Fig. 2. Speed-Torque and speed-Power curves for different $V_{B \max }$ voltages. Colour Legend: standard 2L-VSI (SI) in blue, OEW-FC with $V_{B \max }$ equal to $0.1,0.5,0.8$ and $1 V_{A \max }$ in red, yellow, purple and green respectively.

current rating, looking at Fig. 1, it is evident that for the dual inverter FC topology VSI A and VSI B are series connected, therefore, the current ratings for both of the VSIs must be the same. Fig. 2 shows that both the speed operating range and the CPSR can be significantly improved even when a small voltage $E_{B}$ is allowed. Other drive topologies, operated from a single dc source, which lead to extended speed operating range are considered: the standard 2L-VSI with doubled dc-link voltage, a 2L-VSI with a DC-DC boost converter or an OEW drive connected on the same dc-link. If a standard 2L-VSI with doubled dc-link voltage is considered it is straightforward to observe that the $\mathrm{kVA}$ ratings are doubled as well, the switch count is kept at 6 but the CPSR is not increased. If a DC-DC boost converter topology connected to a dc-link of the same voltage of the on of the VSI A is considered then it will be necessary to add to the system an additional converter with the same kVA ratings of the 2L-VSI and a passive magnetic component, therefore adding volume, cost and losses. The DC-DC boost converter in combination with the $2 \mathrm{~L}-\mathrm{VSI}$ increases the switch count to 8 but is able to provide higher active power than the VSI A of the OEW-FC topology studied but it is not able to extend the CPSR [13] For both the 2L-VSI with doubled dc-link voltage and the DC-DC boost converter plus $2 \mathrm{~L}$-VSI the machine's winding insulation level may need to be improved due to the higher voltage stress. Furthermore, both the topologies can provide a 2 level voltage waveform. The OEW topology with both VSIs connected on the same dc link is able to provide a smaller increment of the speed range, but not as much as it could due to the necessity to apply specific modulations which eliminate the circulating current and result into a reduction of the effective dc-link available at the machine terminals [4], [5]. Regarding the proposed OEW-FC topology, it can be observed that the performance improvement, as a speed range and CPSR increment, strongly depends on the drive 


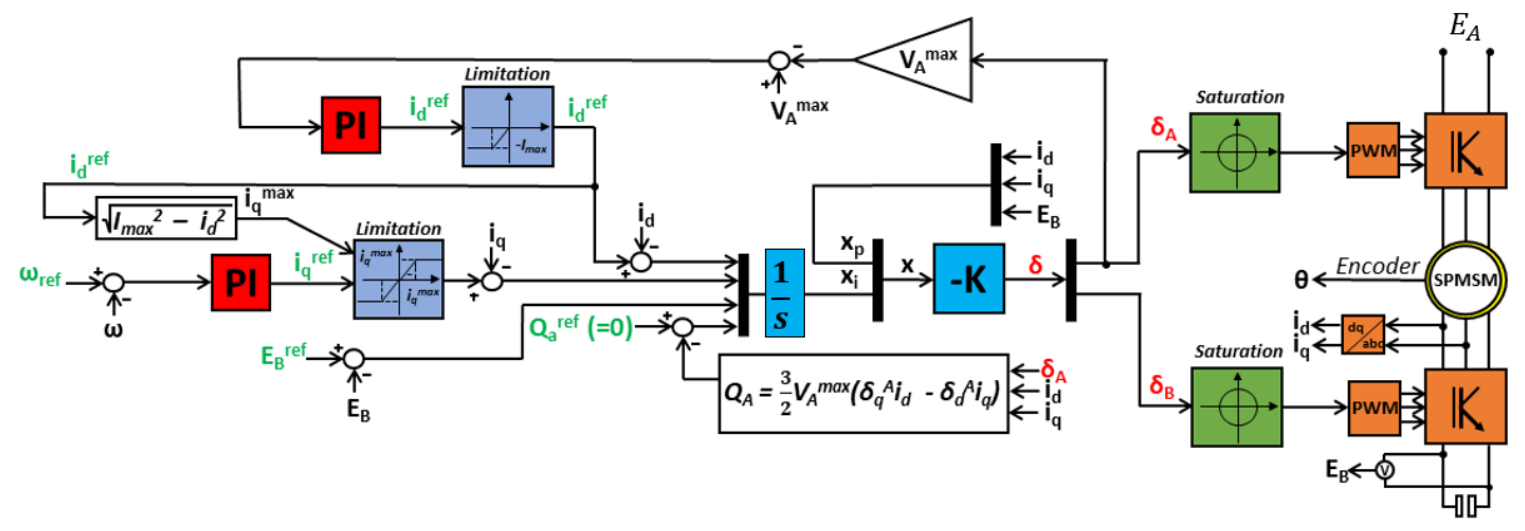

Fig. 3. Block diagram of the control scheme for the OEW-FC SPMSM Drive.

application and the PMSM characteristic current $-\lambda_{m} / L_{d}$. In fact, since the OEW-FC is basically operating only where the PMSM requires reactive power that the FC can supply, depending on the machine flux and anisotropy level it depends the possibility of speed range and CPSR improvement given by the OEW-FC. Furthermore, observing that VSI B is only capable of supplying reactive power the proposed drive is only able to supply the rated kVA of VSI A while an increased CPSR is obtained in combination with a switch count of 12 and a 3 level voltage waveform. Summarizing, the OEW-FC could be a cost-effective solution compared to the other considered topologies if the active power required does not need to be increased over the kVA ratings of VSI A and if an increased CPSR is required, the actual increment has to be evaluated according to the machine characteristic current $-\lambda_{m} / L_{d}$.

\section{State Space Model Formulation And CONTROLlER DESIGN}

\section{A. OEW-FC State Space Model}

The state space model of the OEW-FC dual inverter drive can be obtained by substituting the expression of $v_{d}$ and $v_{q}$ as a function of the duty cycles found in (4) into the machine voltage equations (1). The overall system model is described by the obtained equations united with (5).

$$
\left\{\begin{array}{l}
\frac{d}{d t} i_{d}=-\frac{R_{s}}{L_{s}} i_{d}+w_{e} i_{q}+\frac{E_{A}}{\sqrt{3}} \delta_{d}^{A}-\frac{E_{B}}{\sqrt{3}} \delta_{d}^{B} \\
\frac{d}{d t} i_{q}=-\frac{R_{s}}{L_{s}} i_{q}-w_{e} i_{d}+\frac{E_{A}}{\sqrt{3}} \delta_{q}^{A}-\frac{E_{B}}{\sqrt{3}} \delta_{q}^{B} \\
\frac{d}{d t} E_{B}=\frac{3}{4 C}\left(\delta_{d}^{B} i_{d}+\delta_{q}^{B} i_{q}\right)
\end{array}\right.
$$

As previously described, in order to extend the operating region of the SPMSM the the reactive power supplied by VSI A has to be controlled to zero. The reactive power of the main VSI A $Q_{A}$ can be written as

$$
Q_{A}=\frac{3 E_{A}}{2 \sqrt{3}}\left(\delta_{q}^{A} i_{d}-\delta_{d}^{A} i_{q}\right)
$$

It can be noticed that (11) and (12) are non-linear due to the products between the plant states $\left(i_{d}, i_{q}\right)$ and the control actions $\left(\delta_{d}^{A}, \delta_{q}^{A}, \delta_{d}^{B}, \delta_{q}^{B}\right)$. In order to be able to synthesize a state feedback linear controller a linearized mathematical model of the plant is necessary. Therefore, the OEW-FC SPMSM model represented by (11) and (8) are linearised by calculating the first order partial derivative Jacobian matrices around a specific operating point defined by a reference speed, machine currents and duty cycles $\left[w_{e}^{*}, i_{d}^{*}, i_{q}^{*}, \delta_{d}^{A *}, \delta_{q}^{A *}, \delta_{d}^{B *}, \delta_{q}^{B *}\right]$. The linearized plant in the state space form is presented in (13)

$$
\begin{gathered}
\frac{d}{d t}\left[\begin{array}{c}
i_{d} \\
i_{q} \\
E_{B}
\end{array}\right]=A\left[\begin{array}{c}
i_{d} \\
i_{q} \\
E_{B}
\end{array}\right]+B\left[\begin{array}{l}
\delta_{d}^{A} \\
\delta_{q}^{A} \\
\delta_{d}^{B} \\
\delta_{q}^{B}
\end{array}\right] \\
{\left[\begin{array}{c}
i_{d} \\
i_{q} \\
E_{B} \\
Q_{A}
\end{array}\right]=C\left[\begin{array}{c}
i_{d} \\
i_{q} \\
E_{B}
\end{array}\right]+D\left[\begin{array}{c}
\delta_{d}^{A} \\
\delta_{q}^{A} \\
\delta_{d}^{B} \\
\delta_{q}^{B}
\end{array}\right]}
\end{gathered}
$$

where $A, B, C$ and $D$ are

$$
\begin{gathered}
A=\left[\begin{array}{ccc}
-\frac{R_{s}}{L_{s}} & w_{e}^{*} & -\frac{\delta_{d}^{B *}}{\sqrt{3} L_{s}} \\
-w_{e}^{*} & -\frac{R_{s}}{L_{s}} & -\frac{\delta_{q}^{B *}}{\sqrt{3} L_{s}} \\
\frac{3}{4 C} \delta_{d}^{B *} & \frac{3}{4 C} \delta_{q}^{B *} & 0
\end{array}\right] \\
B=\left[\begin{array}{cccc}
\frac{E_{a}}{\sqrt{3} L_{s}} & 0 & -\frac{E_{B}^{*}}{\sqrt{3} L_{s}} & 0 \\
0 & \frac{E_{a}}{\sqrt{3} L_{s}} & 0 & -\frac{E_{B}^{*}}{\sqrt{3} L_{s}} \\
0 & 0 & \frac{3}{4 C} i_{d}^{*} & \frac{3}{4 C} i_{q}^{*}
\end{array}\right] \\
C=\left[\begin{array}{ccc}
1 & 0 & 0 \\
0 & 1 & 0 \\
0 & 0 & 1 \\
\frac{3 E_{A}}{2 \sqrt{3}} \delta_{q}^{A *} & -\frac{3 E_{A}}{2 \sqrt{3}} \delta_{d}^{A *} & 0
\end{array}\right] \\
D=\left[\begin{array}{cccc}
0 & 0 & 0 & 0 \\
0 & 0 & 0 & 0 \\
0 & 0 & 0 & 0 \\
-\frac{3 E_{A}}{2 \sqrt{3}} i_{q}^{*} & \frac{3 E_{A}}{2 \sqrt{3}} i_{d}^{*} & 0 & 0
\end{array}\right]
\end{gathered}
$$




$$
\begin{aligned}
& \Phi=\left[\begin{array}{cccccccc}
-\frac{R_{s}}{L_{s}} T_{s}+1 & w_{e}^{*} T_{s} & -\frac{\delta_{d}^{B *}}{\sqrt{3} L_{s}} T_{s} & 0 & 0 & 0 & 0 \\
-w_{e} * T_{s} & -\frac{R_{s}}{L_{s}} T_{s}+1 & -\frac{\delta_{q}^{B *}}{\sqrt{3} L_{s}} T_{s} & 0 & 0 & 0 & 0 \\
\frac{3}{4 C} \delta_{d}^{B *} T_{s} & \frac{3}{4 C} \delta_{q} T_{s} & 1 & 0 & 0 & 0 & 0 \\
-T_{s} & 0 & 0 & 1 & 0 & 0 & 0 \\
0 & -T_{s} & 0 & 0 & 1 & 0 & 0 \\
0 & 0 & -T_{s} & 0 & 0 & 1 & 0 \\
-\frac{3 E_{A}}{2 \sqrt{3}} \delta_{q}^{A *} T_{s} & \frac{3 E_{A}}{2 \sqrt{3}} \delta_{d}^{A *} T_{s} & 0 & 0 & 0 & 0 & 1
\end{array}\right] \quad \Gamma=\left[\begin{array}{ccccc}
\frac{E_{a}}{\sqrt{3} L_{s}} T_{s} & 0 & -\frac{E_{B}^{*}}{\sqrt{3} L_{s}} T_{s} & 0 \\
0 & \frac{E_{a}}{\sqrt{3} L_{s}} T_{s} & 0 & -\frac{E_{B}^{*}}{\sqrt{3} L_{s}} T_{s} \\
0 & 0 & \frac{3}{4 C} i_{d}^{*} T_{s} & \frac{3}{4 C} i_{q}^{*} T_{s} \\
0 & 0 & 0 & 0 \\
0 & 0 & 0 & 0 \\
0 & 0 & 0 & 0 \\
\frac{3 E_{A}}{2 \sqrt{3}} i_{q}^{*} T_{s} & -\frac{3 E_{A}}{2 \sqrt{3}} i_{d}^{*} T_{s} & 0 & 0
\end{array}\right] \\
& \boldsymbol{x}=\left[\begin{array}{lllllll}
i_{d} & i_{q} & E_{B} & x_{i_{d}} & x_{i_{q}} & x_{E_{B}} & x_{Q_{A}}
\end{array}\right]^{T} \quad \boldsymbol{u}=\left[\begin{array}{llll}
\delta_{d}^{A} & \delta_{q}^{A} & \delta_{d}^{B} & \delta_{q}^{B}
\end{array}\right]^{T}
\end{aligned}
$$

Once the linearized state space matrices $A, B, C$ and $D$ of the plant have been defined a linear state feedback regulator is designed. Then the overall state space model of the plant and controller can be derived allowing for the gain calculation and controller tuning.

\section{B. OEW-FC Controller design}

Referring to Fig. 3, the inner controller is designed as a state feedback action plus integral controllers placed in the open loop chain for $i_{d}, i_{q}, E_{B}$ and $Q_{A}$ to allow for steady state error elimination. In order to synthesize the controller matrix gain $\mathrm{K}$ the whole plant plus controller state space model has to be derived. The whole system state space model is obtained by state augmentation, i.e. the integral states $\boldsymbol{x}_{\boldsymbol{i}}=\left[\begin{array}{llll}x_{i_{d}} & x_{i_{q}} & x_{E_{B}} & x_{Q_{A}}\end{array}\right]$ are added to the state vector. Finally, the obtained state space model has been discretized according to the Euler method, the resulting discrete OEWFC SPMSM state space model augmented with the controller integral actions is presented in (15) and (10).

$$
\boldsymbol{x}_{k+1}=\Phi \boldsymbol{x}_{k}+\Gamma \boldsymbol{u}_{k}
$$

The system model discretization is a strictly necessary stape to allow for digital implementation of the controller.

Regarding the outer control loop, always referring to Fig. 3 , it is split into a PI speed controller and a PI flux-weakening loop. The voltage references of INV A are used to calculate the required dc link voltage and then used as the input of the feedback-style flux-weakening controller. The reference d-axis current is given by a PI regulator, its output voltage is saturated between zero and the maximum current in the negative d-axis, which is set equal to the maximum machine current $I_{\max }$. The reference q-axis current is modified accordingly. In this work, as previously demonstrated in [9], to reduce the switching losses of VSI B, the floating-capacitor-voltage reference is raised according to the optimal reference voltage necessary to guarantee a unity power factor for VSI A as calculated in (7).

\section{Controller Synthesis}

A convenient way to synthesize the controller is to use a LQR approach [14]. Given a system in the state space form, the optimal LQR consists in the feedback control law (16)

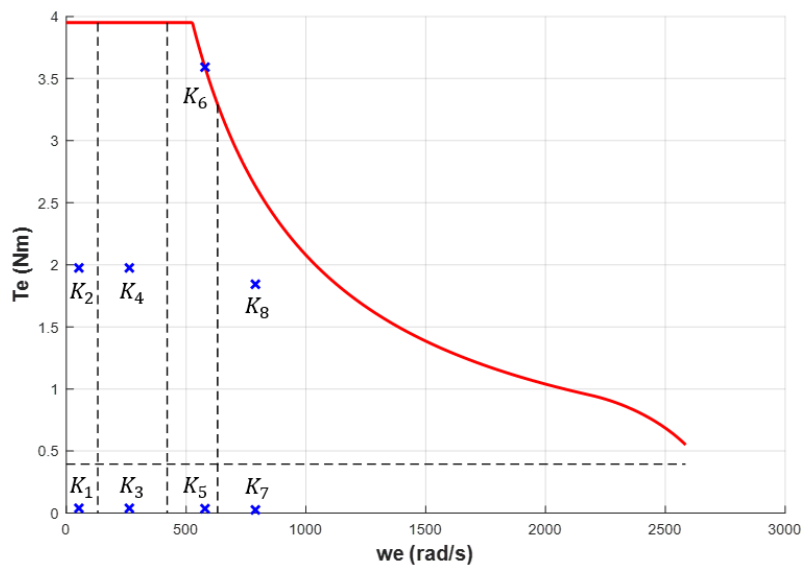

Fig. 4. Speed-Torque curve of the OEW-FC drive. The dashed black lines delimit the gain scheduling regions while the blue cross indicates the respective tuning point, i.e. the operating point on which the plant has been linearised and the controller has been synthesized.

$$
\boldsymbol{u}=-K \boldsymbol{x}
$$

that minimizes the cost $\mathrm{J}$ defined in (17)

$$
J=\int_{0}^{\infty} \boldsymbol{x}^{\prime} Q_{p} \boldsymbol{x}+\boldsymbol{u}_{\boldsymbol{p}}^{\prime} R_{p} \boldsymbol{u}_{\boldsymbol{p}} d t
$$

where $Q_{p} \in \mathbb{R}^{7 x 7}$ and $R_{p} \in \mathbb{R}^{4 x 4}$ are the state and control weighting matrices respectively. It can be shown that this control actions result in a state feedback of the form

$$
\boldsymbol{u}=-K \boldsymbol{x}=-\left[\begin{array}{l}
k_{p} \\
k_{i}
\end{array}\right]\left[\begin{array}{ll}
\boldsymbol{x}_{\boldsymbol{p}} & \boldsymbol{x}_{\boldsymbol{i}}
\end{array}\right]
$$

where $k_{p} \in \mathbb{R}^{4 x 3}$ and $k_{i} \in \mathbb{R}^{4 x 4}$ are the state gains matrix and the integral gains matrix respectively. Due to the nonlinear behaviour of the plant a single feedback gain $K$ able to guarantee stability over the whole speed range could not 

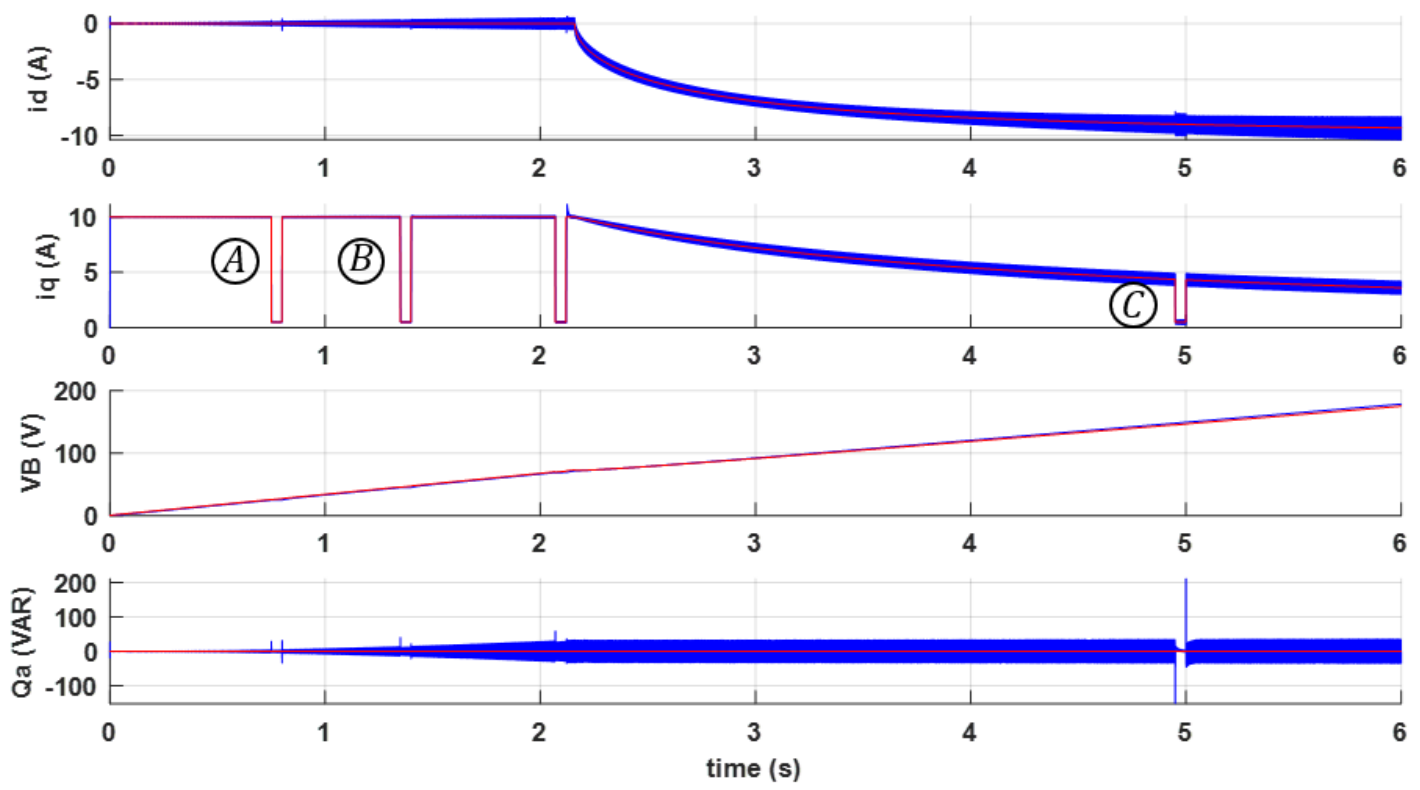

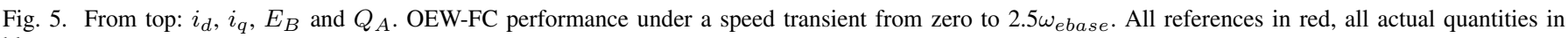
blue.

be identified. Multiple tuning points, as shown in Fig 4, have been selected where the OEW-FC system has been linearised.

The speed-torque plane has been divided into 8 regions, each employing a different feedback gain calculated in its corresponding region tuning point as shown in Fig 4. With the calculated 8 gain matrices $K_{1}, K_{2}, \ldots, K_{8}$, stability can be achieved for the whole operating range on the speed-torque characteristic. It can be noticed that the regions are defined by the lower and upper speed and torque limits. Since the machine considered is an SPMSM the torque is exclusively generated by the q-axis current, therefore, the upper and lower limits of the gain scheduling regions on the torque axis correspond to a lower and upper limit of $i_{q}$. It can be noticed that the gain scheduling regions can be divided in 4 for low torque loads and other 4 for higher values of torque load. Table I summarizes the tuning point of the controller for the 8 regions and their respective upper and lower limits, all quantities are expressed in PU referred to the OEW-FC base speed and the maximum inverter current $I_{\max }$. To allow for a smooth transition from one region to another it is necessary to avoid any discontinuity

TABLE I

Gain Scheduling Tuning Points and Region Boundaries. All QUANTITIES IN PU RESPECT TO THE BASE SPEED AND MAXIMUM MACHINE CURRENT

\begin{tabular}{|c|c|c|c|}
\hline Region & Tuning Point $\left(\boldsymbol{\omega}, \boldsymbol{i}_{\boldsymbol{q}}\right)$ & $\boldsymbol{\omega L \text { Limits }}$ & $\boldsymbol{i}_{\boldsymbol{q}}$ Limits \\
\hline$K_{1}$ & $(0.1,0.01)$ & $(0,0.25)$ & $(0,0.1)$ \\
\hline$K_{2}$ & $(0.1,0.5)$ & $(0,0.25)$ & $(0.1,1)$ \\
\hline$K_{3}$ & $(0.5,0.01)$ & $(0.25,0.8)$ & $(0,0.1)$ \\
\hline$K_{4}$ & $(0.5,0.5)$ & $(0.25,0.8)$ & $(0.1,1)$ \\
\hline$K_{5}$ & $(1.1,0.01)$ & $(0.8,1.2)$ & $(0,0.1)$ \\
\hline$K_{6}$ & $(1.1,0.91)$ & $(0.8,1.2)$ & $(0.1,1)$ \\
\hline$K_{7}$ & $(1.5,0.01)$ & $(1.2,3)$ & $(0,0.1)$ \\
\hline$K_{8}$ & $(1.5,0.47)$ & $(1.2,3)$ & $(0.1,1)$ \\
\hline
\end{tabular}

in the control actions, i.e. the duty cycles of VSI A and B, therefore the integral states are recalculated at every region transition as shown in (19).

$$
\boldsymbol{x}_{\boldsymbol{i k}}=-K_{i}^{-1}\left(k_{p} \boldsymbol{x}_{\boldsymbol{p} \boldsymbol{k}}+\boldsymbol{u}_{\boldsymbol{k}-\mathbf{1}}\right)
$$

Furthermore, to allow for a continuous gain change when crossing from one region to another a small hysteresis band has been introduced and the in order to identify the current region of operation the reference speed and current signals are used being less affected by the measurement noise.

\section{AnAlysis And Simulations Results}

Simulations of the proposed controller have been analysed in Matlab-Simulink and PLECS. The system parameters are reported in Table II. The two VSIs are both switched at $10 \mathrm{kHz}$ with a dead time of $2 \mu \mathrm{s}$. In order to validate the steady state and the transient performances of the proposed controller the drive is tested over the whole speed range. Fig. 5 shows the machine $i_{d}, i_{q}$ currents, the FC voltage $E_{B}$ and the VSI A reactive power $Q_{A}$ over a speed transient that goes from zero to 2.5 times the OEW-FC base speed $\omega_{\text {ebase }}$, that with the dc link voltage selected and the considered machine parameters is $1.67 \mathrm{krpm}$. Furthermore, to validate the stability over different gain scheduling regions four load torque steps

TABLE II

OEW-FC SPMSM DRIVE PARAMETERS

\begin{tabular}{|c|c||c|c|}
\hline $\boldsymbol{P}_{\text {rated }}$ & $1[\mathrm{~kW}]$ & $\boldsymbol{I}_{\boldsymbol{m a x}}$ & $10[\mathrm{~A}]$ \\
\hline $\boldsymbol{w}_{\text {rated }}$ & $1.6[\mathrm{krpm}]$ & $\boldsymbol{p}$ & $3[-]$ \\
\hline $\boldsymbol{R}_{\boldsymbol{s}}$ & $0.24[\Omega]$ & $\boldsymbol{L}_{\boldsymbol{s}}$ & $1.2[\mathrm{mH}]$ \\
\hline $\boldsymbol{\lambda}_{\boldsymbol{m}}$ & $0.08779[\mathrm{~V} \mathrm{~s}]$ & $\boldsymbol{C}$ & $160[\mu \mathrm{F}]$ \\
\hline $\boldsymbol{E}_{\boldsymbol{A}}$ & $80[\mathrm{~V}]$ & $\boldsymbol{E}_{\boldsymbol{B m a x}}$ & $160[\mathrm{~V}]$ \\
\hline
\end{tabular}




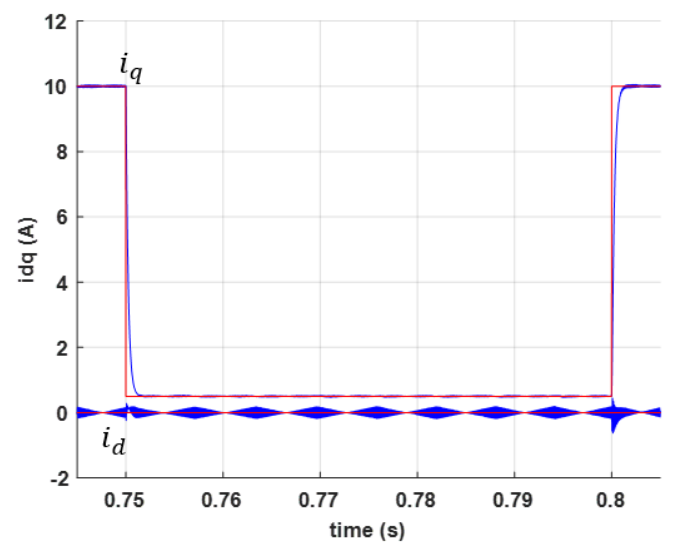

Fig. 6. Load Torque step A of Fig. 5. $i_{d}, i_{q}$ currents dynamics under a load step at $0.25 \omega_{\text {ebase }}$.

at different operating speeds are requested during the speed transient. From Fig. 5 it can be noticed that the proposed controller is still able to obtain the speed range increment associated with the OEW-FC topology, the VSI A reactive power is controlled to zero, hence leading to a unity power factor operation for the battery connected VSI. Figures 6, 7 and 8 are enlargement of the load torque steps A, B and C as indicated in Fig. 5. The load is firstly disconnected and then reconnected to the machine, the step is high enough to cause the $i_{q}$ current to swing from the maximum machine current limit to $0.1 \mathrm{~A}$, therefore causing the transition among two different gain scheduling regions. Fig. 6 and Fig 7 shows a load step at $0.25 \omega_{\text {ebase }}$ and $0.5 \omega_{\text {ebase }}$ respectively. Being below the base speed the $i_{d}$ current is kept at zero while the maximum available $i_{q}$ current equals the maximum machine current $I_{\max }$, in both cases the reference current is tracked while transition between two different gain scheduling regions. Fig 7 shows a load torque step in the flux weakening region of operation of the drive at $2 \omega_{\text {ebase }}$. In all cases it can be noted that the current transient shows satisfying response by still keeping the FC voltage and VSI A reactive power under control.

\section{Vi. CONCLUSIONS}

The dual-inverter topology with a FC bridge feeding an OEW-SPMSM allows for a significant extension of operating speed range and the CPSR compared to the standard 2LVSI. Nevertheless, the controller design and synthesis is not straightforward due to the highly coupled dynamics of the 2 VSIs and the non-linear behaviour of the plant. In this work the state space model of the OEW-FC drive has been derived, a state feedback approach for the internal control loop has been presented and the controller synthesis has been achieved through a LQR to facilitate the use of this promising drive topology.

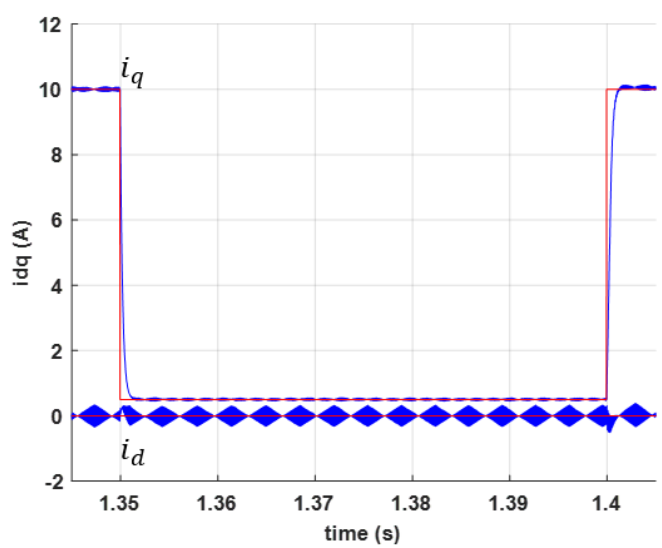

Fig. 7. Load Torque step B of Fig. 5. $i_{d}, i_{q}$ currents dynamics under a load step at $0.5 \omega_{\text {ebase }}$.

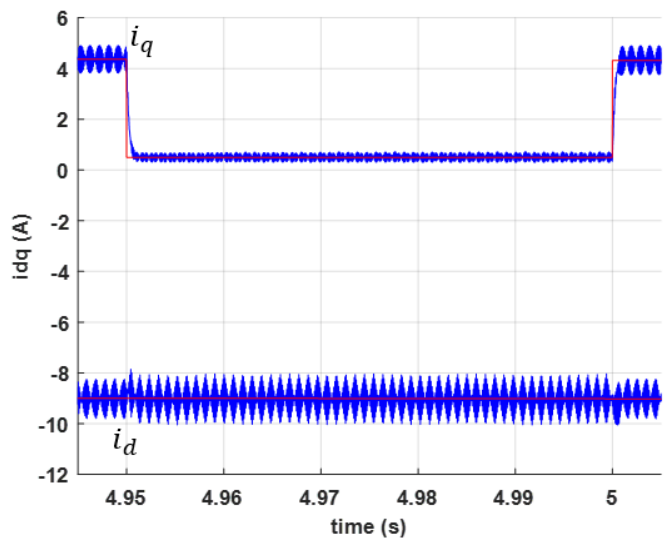

Fig. 8. Load Torque step C of Fig. 5. $i_{d}, i_{q}$ currents dynamics under a load step at $2 \omega_{\text {ebase }}$.

\section{REFERENCES}

[1] H. Stemmler and P. Guggenbach, "Configurations of high-power voltage source inverter drives," in 1993 Fifth European Conference on Power Electronics and Applications, Sept 1993, pp. 7-14 vol.5.

[2] B. Wang, G. Localzo, G. E. Murr, J. Wang, A. Griffo, C. Gerada, and T. Cox, "Overall assessments of dual inverter open winding drives," in 2015 IEEE International Electric Machines Drives Conference (IEMDC), 2015, pp. 1029-1035.

[3] D. Casadei, G. Grandi, A. Lega, C. Rossi, and L. Zarri, "Switching technique for dual-two level inverter supplied by two separate sources," in APEC 07 - Twenty-Second Annual IEEE Applied Power Electronics Conference and Exposition, Feb 2007, pp. 1522-1528.

[4] L. Rovere, A. Formentini, G. L. Calzo, P. Zanchetta, and T. Cox, "Zerosequence voltage elimination for dual-fed common dc-link open-end winding pmsm high-speed starter-generator-part i: Modulation," IEEE Transactions on Industry Applications, vol. 55, no. 6, pp. 7804-7812, 2019.

[5] _ "Zero-sequence voltage elimination for dual-fed common dc-link open-end winding pmsm high-speed starter-generator-part ii: Deadtime hysteresis control of zero-sequence current," IEEE Transactions on Industry Applications, vol. 55, no. 6, pp. 7813-7821, 2019.

[6] D. Pan, F. Liang, Y. Wang, and T. A. Lipo, "Extension of the operating region of an ipm motor utilizing series compensation," IEEE Transactions on Industry Applications, vol. 50, no. 1, pp. 539-548, 2014.

[7] A. Amerise, L. Rovere, A. Formentini, M. Mengoni, L. Zarri, and P. Zanchetta, "Control system for open-end winding surface pm synchronous machines with a floating capacitor bridge," in 2018 IEEE 
Energy Conversion Congress and Exposition (ECCE), 2018, pp. 65856591.

[8] — - "Electric drive based on an open-end winding surface pm synchronous machine with a floating capacitor bridge," IEEE Transactions on Industry Applications, vol. 56, no. 3, pp. 2709-2718, 2020.

[9] A. Amerise, M. Mengoni, L. Zarri, A. Tani, S. Rubino, and R. Bojoi, "Open-end windings induction motor drive with floating capacitor bridge at variable dc-link voltage," IEEE Transactions on Industry Applications, vol. 55, no. 3, pp. 2741-2749, 2019.

[10] S. Chowdhury, P. W. Wheeler, C. Patel, and C. Gerada, "A multilevel converter with a floating bridge for open-end winding motor drive applications," IEEE Transactions on Industrial Electronics, vol. 63, no. 9, pp. 5366-5375, 2016.

[11] S. Chowdhury, P. W. Wheeler, C. Gerada, and C. Patel, "Model predictive control for a dual-active bridge inverter with a floating bridge," IEEE Transactions on Industrial Electronics, vol. 63, no. 9, pp. 55585568, 2016.

[12] Yan Guo, Xiao Wang, H. C. Lee, and Boon-Teck Ooi, "Pole-placement control of voltage-regulated pwm rectifiers through real-time multiprocessing," IEEE Transactions on Industrial Electronics, vol. 41, no. 2, pp. 224-230, 1994.

[13] K. K. Prabhakar, M. Ramesh, A. Dalal, C. U. Reddy, A. K. Singh, and P. Kumar, "Efficiency investigation for electric vehicle powertrain with variable dc-link bus voltage," in IECON 2016 - 42nd Annual Conference of the IEEE Industrial Electronics Society, 2016, pp. 1796-1801.

[14] J. M. B.D.O. Anderson, "Optimal control: Linear quadratic methods," Prentice Hall, Englewood Cliffs, New Jersey, 1989. 